\title{
A case report on symptomatic primary herpetic gingivostomatitis
}

\begin{abstract}
Primary herpetic gingivostomatitis is a common paediatric infection and the causative organism in $90 \%$ of cases is herpes simplex virus type 1, with complications that range from indolent cold sores to dehydration and even life-threatening encephalitis. A nine year old, female presented with a main complaint of difficulty eating due to pain associated with multiple shallow oval oral ulcers. PHG has to be differentiated clinically from acute necrotising ulcerative gingivitis, recurrent HSV infection, herpangia, aphtous ulcers, erythema multiforme, teething, allergic stomatitis and ulcers due to chemotherapy. Management of PHG is palliative and supportive, with symptomatic relief that primarily involves pain management and oral fluids to prevent dehydration until the viral infection subsides, prevention of dehydration, and shortening the duration of lesions even though orolabial herpetic infections are usually self-limiting. The aim of this case study was to highlight a symptomatic case of PHG which required an astute diagnosis and a comprehensive treatment plan with emphasises on the importance for oral health clinicians to have a holistic approach to management of oral disease.
\end{abstract}

Keywords: gingivostomatitis, herpes simplex virus, ulcers, fever
Volume 8 Issue 8 - 2017

\section{Mervyn Turton}

Department of Dentistry, University of the Western Cape, USA

Correspondence: Mervyn Turton, Department of Dentistry, University of the Western Cape, Robert Sobukwe Rd, Bellville, Cape Town, 7535, South Africa, Email mturton@webmail.co.za

Received: November II, 2017 | Published: December 29, 2017
Abbreviations: PHG, primary herpetic gingivostomatitis; HSV, herpes simplex virus

\section{Introduction}

PHG is a common paediatric infection usually seen before 6years of age. ${ }^{1}$ The causative organism in $90 \%$ of cases is HSV type 1 , with complications that range from indolent cold sores ${ }^{1}$ to dehydration ${ }^{2}$ and even life-threatening encephalitis. ${ }^{3}$ For an accurate diagnosis, PHG has to be differentiated clinically from herpetiform apthous stomatitis, recurrent HSV infection, acute necrotising ulcerative gingivitis, allergic stomatitis, erythema multiforme and ulcers due to chemotherapy. PHG is characterised inflammation of the marginal and attached gingivae characterized by erythema, oedema, capillary proliferation and widespread vesicular eruptions affecting vermilion border of lip and labial mucosa, tongue, buccal and vestibular mucosa, hard and soft palate, floor of the mouth, tonsillar and pharyngeal mucosa. $^{3-5}$ The condition is highly contagious ${ }^{3}$ and a diagnosis of PHG is made based on clinical presentation of erythematous gingiva, mucosal haemorrhages, and clusters of small erupted vesicles throughout the mouth in children with symptoms though most children will be asymptomatic. ${ }^{4,5}$ Symptomatic relief primarily involves pain management and oral fluids to prevent dehydration until the viral infection subsides ${ }^{6}$ This case study highlights a symptomatic case of PHG which required an astute diagnosis and a comprehensive treatment plan. It highlights the presentation of PHG and the possible differential diagnosis that could be considered based on current literature. This case study also emphasises the importance for oral health clinicians to have a holistic approach to management of oral disease as treating the patient holistically can bring about positive changes in the persons oral health status, awareness and behaviours.

\section{Case}

A nine year old, female presented with a main complaint of difficulty eating due to pain associated with multiple shallow oval oral ulcers. The patient's mother said her daughter complained of pain 3 days ago and she started eating less due to pain and a gradual lack of appetite. She experienced constant pain in the entire mouth without any burning sensation. The pain became more pronounced when she was eating and had difficulty swallowing. On extra oral examination enlarged submandibular lymph nodes were palpable both on left and right side which were mobile, tender and soft in consistency. Patient appeared weak and dehydrated and had a fever. Her speech was slurred and she had no known allergies and she was HIV negative. On intra oral examination she had an erythematous area surrounding the inner border of the upper and the lower labial mucosa (Figure 1). The marginal and attached gingiva was swollen and inflamed, the gingiva formed tissue tags over the lower primary canines ( 73 and 83) characterized by erythema, oedema, capillary proliferation (Figure 2). Multiple shallow ulcers measuring less than $0.5 \mathrm{~cm}$ were present on the floor of the mouth, tongue lateral borders, the buccal, labial and gingival mucosa which were surrounded by an erythematous area (Figures $3 \& 4$ ). Patient had no oozing from any of the ulcers however they were extremely painful on swallowing or talking. The patient had severe halitosis and a high salivary flow and her teeth had a severe build-up of in plaque and calculus deposits. The lower posterior molars $(74,75,84$ and 85$)$ had tooth decay however the teeth were asymptomatic. 


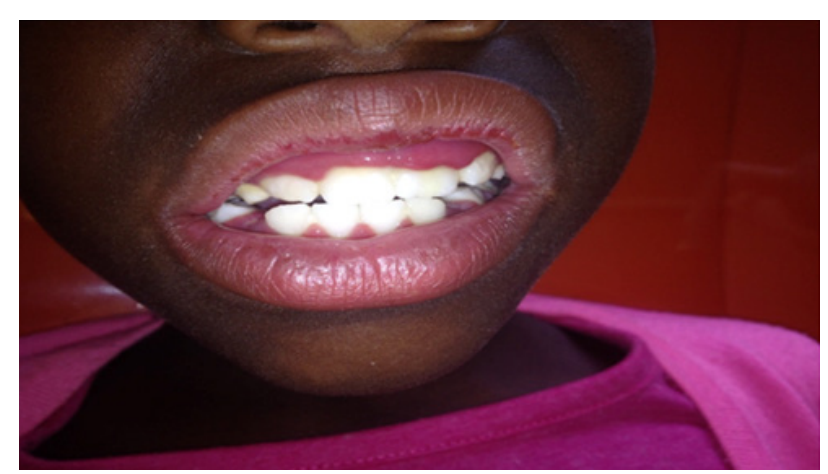

Figure I Upper labial mucosa surrounded by an erythematous area.

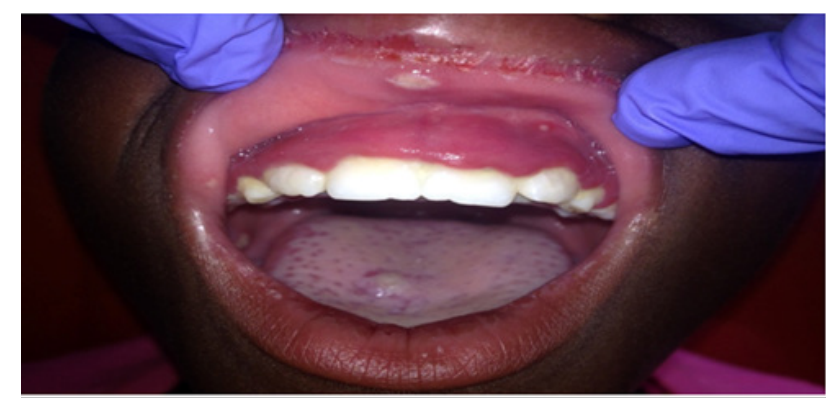

Figure $\mathbf{2}$ Inflammation of the marginal and attached gingivae.

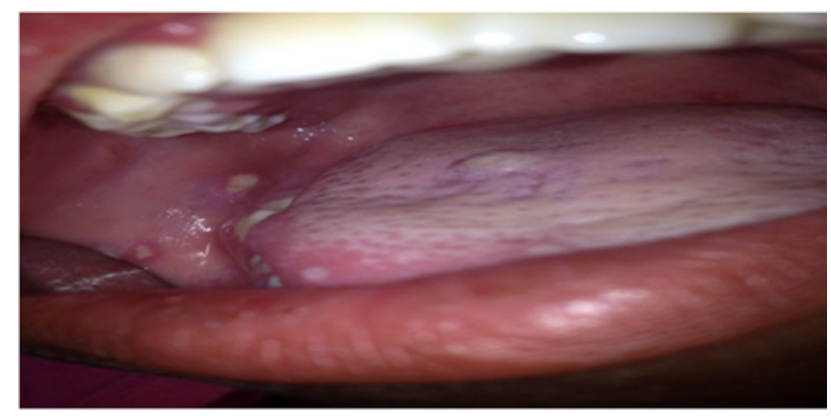

Figure 3 Ulcers on the buccal mucosa and lateral border of the tongue.

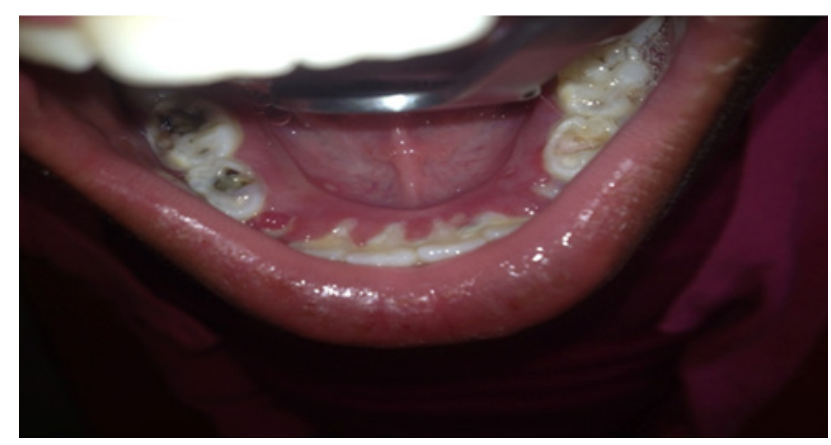

Figure 4 Multiple ulcers on lower gingival mucosa and floor of the mouth.

\section{Management}

Treatment of the patient was initially making her to rinse with an anti-inflammatory and analgesic oral rinse containing benzydamine hydrochloride (Andolex ${ }^{\circledR}$ ) to ease the topical oral pain. Oral rehydration solution was administered as per WHO guidelines. ${ }^{7}$ Andolex oral rinse was dispensed for her to rinse 10minutes before meals as the analgesic and anti-inflammatory effects will reduce the oral pain help her to eat. Paracetamol suspension $5 \mathrm{ml}$ three times daily for 5 days and Metronidazole suspension $5 \mathrm{ml}$ three times daily for 5days was dispensed to be taken after meals. The mother was advised to give her soft diet, copious liquid intake and adequate rest. A recall appointment for 1 weeks' time was given for assessment and further treatment. On presentation for her recall appointment she had regained her health, the ulcers had healed without scarring and the gingiva was less inflamed, her pain and fever had subsided and she was eating and communicating well. Plaque and calculus removal and restoration of the carious teeth was carried out and given comprehensive oral hygiene instruction and advised to only use the oral rinse once daily another reassessment appointment was given for a week later. At her third appointment she had completely recovered and was looking healthy and full of life. An oral examination revealed good oral health. The patient was once again given comprehensive oral hygiene instruction. An alcohol-free $0.05 \%$ sodium fluoride rinse for daily use (ORO-NaF®) was dispensed and her next appointment was scheduled for six months later.

\section{Discussion}

In more than $90 \%$ of PHG cases the causative organism is herpes simplex virus type I and occasionally by herpes simplex virus type II. ${ }^{8}$ The majority of primary infections are asymptomatic or very mild that it goes unobserved. In symptomatic patients the incubation period for PHG is 2-20days, thereafter there is a prodromal phase which is characterised by symptoms which include fever, chills, malaise, irritability, headache, and anorexia. ${ }^{89}$ The acute phase has a rapid onset and is usually characterized by pain, salivation, fetor oris, and submandibular and cervical lymphadenopathy. ${ }^{8,9}$ Examination reveals inflammation of the marginal and attached gingivae characterized by erythema, oedema, capillary proliferation and widespread vesicular eruptions affecting vermilion border of lip and labial mucosa, tongue, buccal and vestibular mucosa, hard and soft palate, floor of the mouth, tonsillar and pharyngeal mucosa. ${ }^{11,12} \mathrm{New}$ vesicles continue to erupt, coalesce, rupture within 24 to 48 hours, and produce shallow, painful, irregular erosions or ulcers circumscribed by a red halo. This continues for 3 to 5 days thereafter healing is without scaring which occurs gradually within 7 to 14 days ${ }^{10-12}$

\section{Differential diagnosis}

PHG has to be differentiated clinically from acute necrotising ulcerative gingivitis, recurrent HSV infection, herpangia, aphtous ulcers, erythema multiforme, teething, allergic stomatitis and ulcers due to chemotherapy ${ }^{8,9}$. ANUG presents with ulcers that are necrotic and punched out seen on the marginal gingiva and interdental papilla which are associated with a peculiar metallic taste, pain, tenderness, profuse salivation. Teeth are have a woody sensation or sensitive to pressure. ${ }^{9,10}$ Recurrent herpetic infection is usually associated with prodromal symptoms of tingling and burning sensation and recurs periodically usually at the vermilion border of the lip, with intraoral lesions seen on the gingiva, palate and alveolar mucosa. ${ }^{8,9}$ Herpangia is a disease caused by the Coxsackie A virus that also commonly causes ulcers in the oral cavity of children. ${ }^{13}$ In herpangina, ulcers are usually isolated to the anterior pillar of the mouth and the soft palate, however in herpetic gingivostomatitis, lesions can be found in these locations, and also on the free and attached gingiva, tongue, labial and buccal mucosa ${ }^{13}$ Apthous ulcers are often associated with prodromal 
symptoms. The ulcers are present on labile mucosa and are round symmetrical and shallow surrounded by erythematous halo. ${ }^{10}$ The presence of target lesions on the skin is highly indicative of erythema multiforme. The lesions are large, irregular, deeper and often bleed, within 2-3days the lesions begin to crust and gingival involvement is rare. ${ }^{8,9}$ Gingivostomatitis must also be differentiated from teething as may be associated with similar symptoms as PHG such as fever and difficulty with eating. ${ }^{14}$ Children start losing maternal antibody protection against the herpes virus at about the same time as the onset of teething thus in some cases PHG could be confused as teething. ${ }^{14}$

Allergic stomatitis is a reaction only occurs at site of contact and includes burning sensation or soreness accompanied by erythema and occasionally formations of vesicles and ulcers and is most accurately diagnosed by patch test results from contact with dental materials, oral hygiene products or foods. ${ }^{9}$ Ulcers due to chemotherapy and recurrent HSV infection can be ruled out by taking appropriate history from the patient. ${ }^{9}$ A diagnosis of PHG can be easily confirmed when patients presents with multiple pin point shallow ulcers present on erythematous base involving the labial mucosa, buccal mucosa, floor of the mouth which are coalesce to form large irregular ulcers with the tissue tags, ${ }^{9-11}$ which is followed by an eruption of oral vesicles, round shallow symmetric oral ulcers, and acute marginal gingivitis. Laboratory tests are usually not required in these cases. In in some cases usually involving adults PHG has a less typical clinical picture, making the diagnosis more difficult. ${ }^{9-11}$ Management of PHG is palliative and supportive, consisting of controlling pain and fever, preventing dehydration, and shortening the duration of lesions even though orolabial herpetic infections are usually self-limiting. ${ }^{9-11}$ Antiviral chemotherapy is available for the treatment of patients at increased risk of complications can be treated with antiviral chemotherapy. ${ }^{9-11}$ Patients should be advised to rest and put on dietary supplements, eat a soft balanced diet, and ensure an adequate intake of fluids, vitamins, and minerals. Adult should be further advised to avoid smoking tobacco products and drinking alcoholic beverages. The use of analgesics, antipyretics, topical anaesthetics and rinsing with an anaesthetic containing mouthwash before each meal, effectively reduces pain during eating.

\section{Conclusion}

PHG in some instances is asymptomatic and even go unnoticed, however in symptomatic cases it can be a very debilitating condition. It is vital for oral health clinicians to be aware of the indicative signs and symptoms of this condition and not to confuse it with other similar conditions on presentation. It is important that a holistic approach to management of oral disease is always adhered to, with the vision that it can bring about positive changes in the persons oral health status, awareness and behaviour.

\section{Funding}

None.

\section{Acknowledgements}

I gratefully acknowledge the patient and her mother who consented to participate in this case report. I also gratefully acknowledge Shizelle Turton for the photography.

\section{Conflicts of interest}

There is no conflict of interest.

\section{References}

1. Kolokotronis A, Doumas S. Herpes simplex virus infection, with particular reference to the progression and complications of primary herpetic gingivostomatitis. Clin Microbiol Infect. 2006;12(3):202-211.

2. Amir J, Harel L, Smetana Z, et al. The natural history of primary herpes simplex type 1 gingivostomatitis in children. Pediatr Dermatol. 1999;16(4):259-263.

3. Sarioglu B, Kose SS, Saritas S, et al. Severe acute disseminated encephalomyelitis with clinical findings of transverse myelitis after herpes simplex virus infection. J Child Neurol. 2014;29(11):1519-1123.

4. Tovaru S, Parlatescu I, Tovaru M, et al. Primary herpetic gingivostomatitis in children and adults. Quintessence Int. 2009;40(2):119-124.

5. Amir J, Harel L, Smetana Z, et al. The natural history of primary herpes simplex type 1 gingivostomatitis in children. Pediatr Dermatol. 1999;16(4):259-263.

6. Faden H. Management of primary herpetic gingivostomatitis in young children. Pediatr Emerg Car. 2006;22(4):268-269.

7. World Health Organization. The Treatment of Diarrhea, A manual for physicians and other senior health workers. WHO Press. Geneva, Switzerland: World Health Organization; 2005.

8. Esmann J. The many challenges of facial herpes simplex virus infection. J Antimicrob Chemother. 2001;47(Suppl T1):17-27.

9. Laller S, Aljehani AAM, Alshadidi AAF, et al. Gingiva in fever blisters in 5year old: Case report. WJPMR. 2017;3(1):279-281.

10. Greenberg MS. Ulcerative, vesicular, and bullous lesions. In:Greenberg MS, Glick M, editors. Burket's oral medicine, diagnosis and treatment. $10^{\text {th }}$ ed. Canada: Decker Intellectual Properties; 2003. 50-84 p.

11. Amir J, Harel L, Smetana Z, et al. The natural history of primary herpes simplex type 1 gingivostomatitis in children. Pediatr Dermatol. 1999;16(4):259-263.

12. Kolokotronis A, Doumas S. Herpes simplex virus infection, with particular reference to the progression and complications of primary herpetic gingivostomatitis. Clin Microbiol Infect. 2006;12(3):202-211.

13. Parrott RH, Wolf SI, Nudelman J, et al. Clinical and laboratory differentiation between herpangina and infectious (herpetic) Gingivostomatitis. Paediatrics. 1954;14(2):122-129.

14. David L King, William Steinhauer, Franklin Garcfa-Godoy, et al. Herpetic gingivostomatitis and teething difficulty in infants. Paediatric Dentistry. 1952;14(2):82-85. 